\title{
Some Fréchet algebras for which the Chern character is an isomorphism
}

\author{
Maarten Solleveld \\ October 2005
}

\begin{abstract}
Using similarities between topological $K$-theory and periodic cyclic homology we show that, after tensoring with $\mathbb{C}$, for certain Fréchet algebras the Chern character provides an isomorphism between these functors. This is applied to prove that the Hecke algebra and the Schwartz algebra of a reductive $p$-adic group have isomorphic periodic cyclic homology.

Later an appendix was added, to deal with infinite direct products of algebras.

Mathematics Subject Classification (2000) 19D55, 19L10, 22E50, 46L80
\end{abstract}

Let $X$ be a smooth manifold. The classical Chern character is a map that assigns to a vector bundle on $X$ a class in the even De Rham cohomology of $X$. This extends naturally to a ring homomorphism

$$
\mathrm{Ch}: K^{*}(X) \rightarrow H_{D R}^{*}(X)
$$

More or less since the beginning of topological $K$-theory $[\mathrm{AH}$ ] it has been known that if $X$ is compact this yields an isomorphism of $\mathbb{Z} / 2 \mathbb{Z}$-graded algebras

$$
\mathrm{Ch} \otimes \mathrm{id}: K^{*}(X) \otimes \mathbb{R} \rightarrow H_{D R}^{*}(X)
$$

However for noncompact $X$ the graded vector spaces in (2) are not necessarily isomorphic. This is because the $K$-theory of $X$ is not defined directly: one first has to take the one-point compactification of $X$, then determine the $K$-groups of that space, and finally take the quotient by a suitable subgroup isomorphic to $K_{*}$ (point). In other words topological $K$-theory, contrarily to De Rham cohomology, is similar to singular (or Čch) cohomology with compact support.

If we extend our coefficients from $\mathbb{R}$ to $\mathbb{C}$ then both sides of (2) can be expressed in terms of the Fréchet-algebra $C^{\infty}(X)$ of complex-valued smooth functions on $X$ :

$$
\begin{aligned}
K^{*}(X) & \cong K_{*}\left(C^{\infty}(X)\right) \\
H_{D R}^{*}(X ; \mathbb{C}) & \cong H_{*}^{D R}\left(C^{\infty}(X)\right)
\end{aligned}
$$

Here (3) follows from the smooth version of the Serre-Swan theorem, while (4) is a consequence of the canonical identification

$$
\Omega^{*}(X ; \mathbb{C})=\Omega_{*}\left(C^{\infty}(X)\right)
$$

of the De Rham complex over $X$ with the exterior algebra of $C^{\infty}(X)$.

From the above we get an isomorphism

$$
\mathrm{Ch} \otimes \mathrm{id}: K_{*}(A) \otimes \mathbb{C} \rightarrow H_{*}^{D R}(A)
$$

for $A=C^{\infty}(X)$. Clearly it is desirable to extend (6) to other (noncommutative) Fréchet algebras $A$. Indeed this is a well-studied subject in noncommutative geometry, and strong results have been achieved. The first complication on this path is that, although it is always defined, De Rham 
(co)homology behaves well mainly for commutative algebras. Therefore we must put another functor on the right hand side of (6), and the best choice turns out to be the topological periodic cyclic homology $H P_{*}$. This was justified by Connes, who showed Con that in the above case we have

$$
H P_{*}\left(C^{\infty}(X)\right) \cong H_{*}^{D R}\left(C^{\infty}(X)\right)
$$

Cyclic homology is intimately related to Hochschild homology, analogous to the relation between (4) and (5). Indeed to derive (7) Connes first proved a smooth version of the (algebraic) HochschildKostant-Rosenberg theorem :

$$
H H_{*}\left(C^{\infty}(X)\right) \cong \Omega^{*}(X ; \mathbb{C})
$$

Before we continue we have to specify in exactly which category of algebras we wish to work, because this matters for the definitions and properties of the functors $K_{*}$ and $H P_{*}$. We use the largest that seems reasonable (at present).

Recall that an $m$-algebra is a complete locally convex algebra whose topology is defined by a family of submultiplicative seminorms. It can be shown that $m$-algebras are precisely the projective limits of Banach algebras, and in particular every $C^{*}$-algebra is an $m$-algebra. Fréchet $m$-algebras can be described as those $m$-algebras for which a countable number of seminorms suffices to define the topology, or equivalently as those Fréchet algebras whose seminorms may be taken submultiplicative. Unless explicitly stated otherwise, by the (topological) tensor product of two $m$-algebras we will always mean the projective tensor product, which is also an $m$-algebra.

The $K$-theory of $m$-algebras was defined by Phillips Phi] (for Fréchet $m$-algebras) and by Cuntz Cun2, as a special case of a bivariant functor on $m$-algebras. It satisfies the following properties:

1. additivity: $K_{i}\left(\prod_{n=1}^{\infty} A_{n}\right) \cong \prod_{n=1}^{\infty} K_{i}\left(A_{n}\right)$

2. stability: $K_{i}\left(M_{n}(A)\right) \cong K_{i}(A)$

3. 2-periodicity: $K_{i+2}(A) \cong K_{i}(A)$

4. diffeotopy-invariance: if $\left(\phi_{t}\right)_{t \in[0,1]}$ is a diffeotopy of $m$-algebra homomorphisms then $K_{i}\left(\phi_{0}\right)=$ $K_{i}\left(\phi_{1}\right)$

5. excision: if $I$ is an ideal of $A$ then there exists an exact hexagon

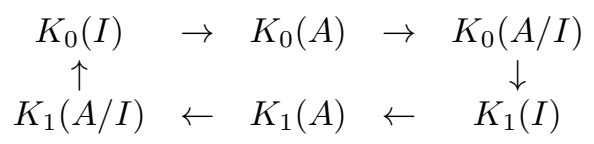

6. continuity: $K_{i}\left(\lim _{n \rightarrow \infty} A_{n}\right) \cong \lim _{n \rightarrow \infty} K_{i}\left(A_{n}\right)$, at least for Banach algebras

Of course this list is by no means exhaustive, for example stability as above is just a weak version of the Morita-invariance of $K$-theory.

Periodic cyclic (co-)homology was first defined by Connes [Con for general locally convex algebras, and he showed directly that it is invariant under diffeotopies. This homology theory satisfies 3 by construction, while 1 and 2 follow from the well known corresponding properties of Hochschild homology. Cuntz Cun2 proved that there exists a unique functorial Chern character on the category of $m$-algebras

$$
\operatorname{ch}: K_{*} \rightarrow H P_{*}
$$

which is compatible with $1-4$.

However there are some problems with excision for $H P_{*}$, and they stem from the fact that a closed subspace of a topological vector space does not always possess a closed complement. 
Therefore we restrict ourselves to admissible extensions of $m$-algebras, i.e. those short exact sequences $0 \rightarrow A \rightarrow B \rightarrow C \rightarrow 0$ which admit a continuous linear splitting $C \rightarrow B$. Equivalently we can require that there exists a continuous projection of $B$ onto $A$. In the same spirit we call an ideal $I$ of $A$ admissible if the associated sequence $0 \rightarrow I \rightarrow A \rightarrow A / I \rightarrow 0$ is.

Theorem 1. Let $0 \rightarrow A \rightarrow B \rightarrow C \rightarrow 0$ be an admissible extension of $m$-algebras. Then excision holds for $H P_{*}$ and the various Chern characters make a commutative diagram

$$
\begin{array}{ccccccccccc}
K_{1}(A) & \rightarrow & K_{1}(B) & \rightarrow & K_{1}(C) & \rightarrow & K_{0}(A) & \rightarrow & K_{0}(B) & \rightarrow & K_{0}(C) \\
\downarrow & & \downarrow & & \downarrow & & \downarrow & & \downarrow & & \downarrow \\
H P_{1}(A) & \rightarrow & H P_{1}(B) & \rightarrow & H P_{1}(C) & \rightarrow & H P_{0}(A) & \rightarrow & H P_{0}(B) & \rightarrow & H P_{0}(C)
\end{array}
$$

Moreover if $\eta: K_{0}(C) \rightarrow K_{1}(A)$ and $\partial: H P_{0}(C) \rightarrow H P_{1}(A)$ are the connecting maps, then ch $\circ \eta=2 \pi i \partial \circ c h$.

Proof. The excision part is due to Cuntz [Cun1], while Nistor [Nis1] showed that the Chern character commutes with the index maps and is compatible with the connecting maps, in the specified sense.

Neither is $H P_{*}$ continuous in general, because inductive limits and projective tensor products do not commute. On the other hand, inductive limits $d o$ commute with inductive tensor products, so the periodic cyclic homology $H P_{*}^{\prime}$ based on this tensor product has a better chance of being continuous. Indeed Brodzki and Plymen BP1] showed that if there exists an $N \in \mathbb{N}$ such that $H H_{i}^{\prime}\left(A_{n}\right)=0 \forall i>N$, then

$$
H P_{i}^{\prime}\left(\lim _{n \rightarrow \infty} A_{n}\right) \cong \lim _{n \rightarrow \infty} H P_{i}^{\prime}\left(A_{n}\right)
$$

However, as long as we are not working with nuclear Fréchet algebras, for which there is only one topological tensor product, excision remains a bit of a problem for this cyclic theory.

Now let us define the category $\mathcal{C}$ of algebras that we are going to study :

Definition 2. The category $\mathcal{C}$ is a full subcategory of the category of $m$-algebras. Its objects are those $m$-algebras $A$ for which the Chern character induces an isomorphism

$$
c h \otimes \mathrm{id}: K_{*}(A) \otimes \mathbb{C} \rightarrow H P_{*}(A) \otimes_{\mathbb{C}} \mathbb{C}=H P_{*}(A)
$$

Corollary 3. The category $\mathcal{C}$ is stable, closed under diffeotopy-equivalences and under finite direct sums. It contains all algebras of the type $C^{\infty}(X)$, where $X$ is a compact smooth manifold.

If $0 \rightarrow A \rightarrow B \rightarrow C \rightarrow 0$ is an admissible extension of $m$-algebras and two elements of $\{A, B, C\}$ are objects of $\mathcal{C}$, then so is the third.

Proof. We only prove the last statement, everything else following directly from the above remarks. Tensor the commutative diagram of Theorem 1 with $\mathbb{C}$. If for example (the other cases being similar) we know that $\{A, C\} \subset \mathrm{Ob}(\mathcal{C})$, then first consider the diagram obtained by deleting the column containing $H P_{1}(B)$. The five lemma shows that $K_{0}(B) \otimes \mathbb{C} \cong H P_{0}(B)$. Likewise, if we retain this column but delete the one with $H P_{0}(B)$ then we deduce that also $K_{1}(B) \otimes \mathbb{C} \cong H P_{1}(B)$, so that indeed $B \in \mathrm{Ob}(\mathcal{C})$.

This last property can also be formulated by saying that $\mathcal{C}$ is closed under taking extensions, ideals and quotients. This can easily be extended to longer sequences of $m$-algebras.

Lemma 4. Consider an increasing sequence

$$
0=I_{0} \subset I_{1} \subset \cdots \subset I_{n} \subset I_{n+1}=A
$$


of admissible ideals of $A$. If all the quotients $I_{m} / I_{m-1}$ are objects of $\mathcal{C}$, then so are all the $I_{m}$ themselves. Similarly let

$$
0 \rightarrow B_{1} \rightarrow B_{2} \rightarrow \cdots \rightarrow B_{n} \rightarrow 0
$$

be an admissible exact sequence of $m$-algebras. If all but one of the $B_{i}$ belong to $\mathcal{C}$, then so does the last.

Proof. Consider the admissible extensions

$$
\begin{aligned}
& 0 \rightarrow \quad I_{m-1} \quad \rightarrow \quad I_{m} \rightarrow \quad \rightarrow \quad I_{m} / I_{m-1} \quad \rightarrow \quad 0 \\
& 0 \rightarrow \operatorname{im}\left(B_{m-1} \rightarrow B_{m}\right) \rightarrow B_{m} \rightarrow \operatorname{im}\left(B_{m} \rightarrow B_{m+1}\right) \rightarrow 0
\end{aligned}
$$

They degenerate for $m=1$, so the lemma follows from Corollary 3, with induction to $n$.

From now on let $G$ be a finite group acting by diffeomorphisms on $X$, which is assumed second countable but not necessarily compact. Wassermann Was2 extended Connes' result (7) to this equivariant noncompact setting by showing that

$$
H P_{*}\left(C^{\infty}(X)^{G}\right) \cong H P_{*}\left(C^{\infty}(X)\right)^{G} \cong H_{*}^{D R}\left(C^{\infty}(X)\right)^{G}
$$

By (4) the right hand side can be identified with the $G$-invariant part $H_{D R}^{*}(X ; \mathbb{C})^{G}$ of the De Rham cohomology of $X$, and Grothendieck proved in Corollaire 5.2.3 of Gro2 that this in turn is naturally isomorphic to $H^{*}(X / G ; \mathbb{C})$, the cohomology of the constant sheaf $\mathbb{C}$ over the orbifold $X / G$. Since $C^{\infty}(X)^{G}$ is by definition the algebra of smooth functions on $X / G$, (10) can be restated by saying that (17) also holds for orbifolds that are quotients of manifolds by finite groups.

A related interesting algebra is the crossed product $C^{\infty}(X) \rtimes G$. To formulate the relevant results we introduce

$$
\hat{X}:=\{(g, x) \in G \times X: g x=x\}
$$

with the $G$-action

$$
g \cdot\left(g^{\prime}, x\right)=\left(g g^{\prime} g^{-1}, g x\right)
$$

The space $\hat{X} / G$ is called the extended quotient of $X$ by $G$. It is a disjoint union of orbifolds, one for each conjugacy class in $G$, and (from a homological point of view) well-suited to deal with singularities of the group action. Brylinski $[\mathrm{Bry}]$ proved that

$$
\begin{aligned}
& H P_{*}\left(C^{\infty}(X) \rtimes G\right) \cong\left(H_{D R}^{*}(\hat{X} ; \mathbb{C})\right)^{G} \\
& H H_{*}\left(C^{\infty}(X) \rtimes G\right) \cong\left(\Omega^{*}(\hat{X} ; \mathbb{C})\right)^{G}
\end{aligned}
$$

By the above result of Grothendieck, (11) is isomorphic to $H^{*}(\hat{X} / G ; \underline{\mathbb{C}})$. Baum and Connes [BC] constructed (for compact $X$ ) an equivariant Chern character

$$
\mathrm{Ch}_{G}: K_{*}\left(C^{\infty}(X) \rtimes G\right) \cong K_{G}^{*}(X) \rightarrow\left(H_{D R}^{*}(\hat{X} ; \mathbb{C})\right)^{G}
$$

and proved that it becomes an isomorphism after tensoring with $\mathbb{C}$. So in this case it has not only been known for long that $K_{*} \otimes \mathbb{C}$ and $H P_{*}$ agree, they have also been determined in geometrical terms.

By the way, the isomorphisms (10) till (13) also hold for the algebra $C_{c}^{\infty}(X)$ of compactly supported smooth functions on $X$, provided that one takes De Rham cohomology with compact support everywhere. See $[\mathrm{BN}]$ for these and more general results on the cyclic homology of algebras associated to orbifolds. 
But things cannot always be this nice. In Was2 it was noticed that, even for compact $X$,

$$
H H_{*}\left(C^{\infty}(X)^{G}\right) \text { and } \Omega^{*}(X ; \mathbb{C})^{G}
$$

are not isomorphic in general.

If $\mathbb{C}^{N}=\mathbb{C} G$ is the regular representation of $G$, then we can endow $C^{\infty}(X ; \operatorname{End}(\mathbb{C} G))=$ $C^{\infty}(X) \otimes \operatorname{End}(\mathbb{C} G)$ with the diagonal $G$-action, and it is well-known that

$$
C^{\infty}(X) \rtimes G \cong C^{\infty}(X ; \operatorname{End}(\mathbb{C} G))^{G}=M_{N}\left(C^{\infty}(X)\right)^{G}
$$

In our main theorem will show that algebras of this type (and some others as well) belong to the category $\mathcal{C}$ defined above. Note that this does not contradict the remark after equation (2), since over there we were actually dealing with the $C^{*}$-algebra $C_{0}(X)$, which is quite different from $C^{\infty}(X)$.

To include manifolds with boundary we recall the following conventions :

Definition 5. Let $Y \subset X$ be arbitrary subsets of a smooth manifold $M$, and let $V$ be a complex vector space.

$$
\begin{aligned}
C^{\infty}(X) & :=\left\{\left.f\right|_{X}: f \in C^{\infty}(U) \text { for some open } U, X \subset U \subset M\right\} \\
C_{0}^{\infty}(X, Y) & :=\left\{f \in C^{\infty}(X):\left.f\right|_{Y}=0\right\} \\
C_{0}^{\infty}(X, Y ; V) & :=C_{0}^{\infty}(X, Y) \otimes V
\end{aligned}
$$

Theorem 6. Let $X$ be a smooth manifold with boundary, $N \in \mathbb{N}$ and consider the $m$-algebra $A=C^{\infty}\left(X ; M_{N}(\mathbb{C})\right)=M_{N}\left(C^{\infty}(X)\right)$. Suppose that a finite group $G$ acts on $A$ by

$$
g \cdot a(x)=u_{g}(x) a\left(\alpha_{g}^{-1} x\right) u_{g}^{-1}(x)
$$

where $\alpha_{g}$ is a diffeomorphism of $X$ and $u_{g} \in A$. Then $A^{G} \in \mathrm{Ob}(\mathcal{C})$, and $K_{*}\left(A^{G}\right)$ is a finitely generated abelian group whenever $X$ is compact.

First we prove a special case, an equivariant version of the Poincaré lemma :

Lemma 7. In the setting of Theorem [6, suppose that $X$ is G-equivariantly contractible to a point $x_{0} \in X$. Then $A^{G}$ is diffeotopy-equivalent to its fiber $\operatorname{End}_{G}\left(\mathbb{C}^{N}\right)$ over $x_{0}$. In particular $K_{*}\left(A^{G}\right)=$ $K_{0}\left(A^{G}\right)$ is a free abelian group of finite rank and $A^{G} \in \mathrm{Ob}(\mathcal{C})$.

Proof. Our main task is to adjust the $u_{g}$ suitably. Since $X$ is contractible we can find for every $g \in G$ a function $f_{g} \in C^{\infty}(X)$ such that $f_{g}^{-N}=\operatorname{det}\left(u_{g}\right)$. The $G$-action does not change when we replace $u_{g}$ with $f_{g} u_{g}$, so we may assume that $\operatorname{det}\left(u_{g}\right) \equiv 1, \forall g \in G$. The premise that (15) is a group action guarantees that there is a smooth function $\lambda: G \times G \times X \rightarrow \mathbb{C}$ such that

$$
u_{g}(x) u_{h}\left(\alpha_{g}^{-1} x\right)=\lambda(g, h, x) u_{g h}(x)
$$

Taking determinants we see that in fact $\lambda(g, h, x)^{N} \equiv 1$, so $\lambda$ does not depend on $x \in X$. All the elements of $\alpha(G)$ fix $x_{0}$, so the fiber $V_{0}=\mathbb{C}^{N}$ over that point is a projective $G$-representation $\left(\pi_{0}, V_{0}\right)$. Thus we are in a position to apply Schur's theorem $[\mathbf{S c h}$, which says that there exists a finite central extension $G^{*}$ of $G$ such that $\pi_{0}$ lifts to a representation of $G^{*}$. This lift only involves scalar multiples of the $u_{g}\left(x_{0}\right)$, so it immediately extends to $X$. Then (16) becomes the cocycle relation

$$
u_{g h}(x)=u_{g}(x) u_{h}\left(\alpha_{g}^{-1} x\right)
$$

Notice that still $A^{G^{*}}=A^{G}$, so without loss of generality we can replace $G$ by $G^{*}$. 
Now we want to make the $u_{g}(x)$ independent of $x \in X$. Wassermann Was1 indicated how this can be done in the continuous case, and his argument can easily be adapted to our smooth setting. The crucial observation, first made by Rosenberg $\left[\operatorname{Ros}\right.$, is that $A^{G}$ can be rewritten as the image of an idempotent in a larger algebra. This idempotent can then be deformed to one independent of $x$.

Indeed, let $A \rtimes_{\alpha} G$ be the crossed product of $A$ and $G$ with respect to the action $\alpha$ of $G$ on $X$, and $\left(r_{t}\right)_{t \in[0,1]}$ a smooth $G$-equivariant contraction from $X$ to $x_{0}$. (For smooth manifolds the existence of a continuous contraction implies the existence of a smooth one.) Define

$$
p_{t}(x):=|G|^{-1} \sum_{g \in G} u_{g}\left(r_{t} x\right) g
$$

Then $p_{t} \in A \rtimes_{\alpha} G$ is an idempotent by (17), and by [Ros]

$$
\phi_{1}: A^{G} \rightarrow p_{1}\left(A \rtimes_{\alpha} G\right) p_{1}: \sigma \rightarrow p_{1} \sigma p_{1}
$$

is an isomorphism of Fréchet algebras. Clearly the idempotents $p_{t}$ are all homotopic, so they are conjugate in the Banach completion $C\left(X ; M_{N}(\mathbb{C})\right) \rtimes_{\alpha} G$ of $A \rtimes_{\alpha} G$. Moreover the standard argument for this, as for example in Proposition 4.3.2 of Bla, shows that $p_{0}$ and $p_{1}$ are conjugate by an element of $A \rtimes_{\alpha} G$. Alternatively we can use the stronger result that homotopic idempotents in Fréchet $m$-algebras are conjugate, but this statement is vastly more difficult to prove than its Banach algebra version, cf. [Phi], Lemma 1.12 and Lemma 1.15. In any case, we have

$$
A^{G} \cong p_{1}\left(A \rtimes_{\alpha} G\right) p_{1} \cong p_{0}\left(A \rtimes_{\alpha} G\right) p_{0} \cong C^{\infty}\left(X ; \operatorname{End}_{\mathbb{C}}\left(V_{0}\right)\right)^{G}
$$

To this last algebra we can apply the obvious diffeotopy $\sigma \rightarrow \sigma \circ r_{t}$. This shows that $A^{G}$ is diffeotopy-equivalent to its fiber $\operatorname{End}_{G}\left(V_{0}\right)$ over $x_{0}$, and the remaining statements on $K_{*}\left(A^{G}\right)$ and $H P_{*}\left(A^{G}\right)$ follow from the semisimplicity of the finite dimensional algebra $\operatorname{End}_{G}\left(V_{0}\right)$.

To make full use of the proof of this lemma we have to study certain ideals in such algebras as well :

Lemma 8. Let $U \subset \mathbb{R}^{n}$ be an open bounded star-shaped set. The m-algebra $C_{0}^{\infty}\left(\mathbb{R}^{n}, \mathbb{R}^{n} \backslash U\right)$ belongs to $\mathcal{C}$ and

$$
K_{*}\left(C_{0}^{\infty}\left(\mathbb{R}^{n}, \mathbb{R}^{n} \backslash U\right)\right) \cong H_{c}^{*}\left(\mathbb{R}^{n}\right) \cong \mathbb{Z}
$$

is concentrated in degree $n$.

Proof. Clearly we may assume that 0 is the center of $U$. Let $P$ be the point of the $n$ sphere corresponding to infinity under the stereographic projection $S^{n} \rightarrow \mathbb{R}^{n}$. By assumption $C_{0}^{\infty}\left(\mathbb{R}^{n}, \mathbb{R}^{n} \backslash U\right) \cong C_{0}^{\infty}\left(S^{n}, Y\right)$ for some closed neighborhood $Y$ of $P$, and we will show that the latter algebra is diffeotopy-equivalent to $C_{0}^{\infty}\left(S^{n}, P\right)$. Let $\left(r_{t}\right)_{t \in[0,1]}$ be a diffeotopy of smooth maps $S^{n} \rightarrow S^{n}$ such that

1. $\forall t: r_{t}(P)=P$ and $r_{t}(Y) \subset Y$

2. a neighborhood of $-P$ is fixed pointwise by all $r_{t}$

3. $r_{1}=\operatorname{id}_{S^{n}}$ and $r_{0}(Y)=P$

To construct such maps, we can require that $r_{t}$ stabilizes every geodesic from $-P$ to $P$ and declare that furthermore $r_{t}(Q)$ depends only on $t$ and on the distance from $Q$ to $P$. Then we only have 
to pick a suitable smooth function of $t$ and this distance. Given this, consider the $m$-algebra homomorphisms

$$
\begin{aligned}
\phi: C_{0}^{\infty}\left(S^{n}, P\right) & \rightarrow C_{0}^{\infty}\left(S^{n}, Y\right): f \rightarrow f \circ r_{0} \\
i: C_{0}^{\infty}\left(S^{n}, Y\right) & \rightarrow C_{0}^{\infty}\left(S^{n}, P\right): f \rightarrow f
\end{aligned}
$$

By construction $\phi \circ i$ and $i \circ \phi$ are diffeotopic to the respective identity maps on $C_{0}^{\infty}\left(S^{n}, Y\right)$ and $C_{0}^{\infty}\left(S^{n}, P\right)$, so these algebras are indeed diffeotopy-equivalent. Thus we reduced our task to calculating the $K$-groups and periodic cyclic homology of $C_{0}^{\infty}\left(S^{n}, P\right)$. Fortunately there is an obvious split extension

$$
0 \rightarrow C_{0}^{\infty}\left(S^{n}, P\right) \rightarrow C^{\infty}\left(S^{n}\right) \rightarrow \mathbb{C} \rightarrow 0
$$

which by Corollary 3 consists entirely of $m$-algebras in the category $\mathcal{C}$. It is well known that

$$
K_{*}\left(C^{\infty}\left(S^{n}\right)\right) \cong K^{*}\left(S^{n}\right) \cong \mathbb{Z}^{2}
$$

with one copy of $\mathbb{Z}$ in degree 0 and the other in degree $n$. Since $K_{*}(\mathbb{C})=K_{0}(\mathbb{C}) \cong \mathbb{Z}$ the lemma follows from the excision property of the $K$-functor.

Proof of Theorem 6. All our arguments will depend on the existence of a specific cover of $X$. To construct it we use a theorem of Illman IIl, which states that $X$ has a smooth equivariant triangulation. In slightly more down-to-earth language this means (among others) that there exists a countable, locally finite simplicial complex $\Sigma$ in a finite dimensional orthogonal representation space $V$ of $G$, and a $G$-equivariant homeomorphism $\psi: \Sigma \rightarrow X$. Moreover $\psi$ is smooth as a map from a subset of $V$ to $X$, and its restriction to any simplex $\sigma$ of $\Sigma$ is an embedding.

For every such $\sigma$ we put

$$
U_{\sigma}^{\prime}:=\left\{v \in \Sigma: d(v, \sigma) \leq r_{\sigma}\right\}
$$

where $d$ is the Euclidean distance in $V$. We require that the radius $r_{\sigma}$ depends only on the $G$-orbit of $\sigma$ and that $r_{\tau}>r_{\sigma}>0$ if $\tau$ is a face of $\sigma$. The orthogonality of the action of $G$ on $V$ guarantees that

$$
g U_{\sigma}^{\prime}=U_{g \sigma}^{\prime} \quad \text { and } \quad U_{\sigma}^{\prime} \cap U_{\tau}^{\prime} \subset U_{\sigma \cap \tau}^{\prime}
$$

if we take our radii small enough. Let $D_{\sigma}^{\prime}$ be the union, over all faces $\tau$ of $\sigma$, of the $U_{\tau}^{\prime}$, and $G_{\sigma}$ the stabilizer of $\sigma$ in $G$. From the above we deduce that $U_{\sigma}^{\prime} \backslash D_{\sigma}^{\prime}$ is $G_{\sigma}$-equivariantly retractible to $\sigma \backslash D_{\sigma}^{\prime}$.

Now we abbreviate $U_{\sigma}:=\psi\left(U_{\sigma}^{\prime}\right)$ and $D_{\sigma}:=\psi\left(D_{\sigma}^{\prime}\right)$, so that $\mathcal{U}:=\left\{U_{\sigma}: \sigma\right.$ simplex of $\left.\Sigma\right\}$ is a closed $G$-equivariant cover of $X$. Let $X_{m}$ be the union of all those $U_{\sigma}$ for which $m+\operatorname{dim} \sigma \leq \operatorname{dim} X$. It is a closed subvariety (with boundary and corners) of $X$ and it is stable under the action of $G$. Define the following $G$-stable ideals of $A$ :

$$
I_{m}:=\left\{a \in A:\left.a\right|_{X_{m}}=0\right\}
$$

By Théorème IX.4.3 of [Tou]

$$
0 \rightarrow I_{m} \rightarrow A=C^{\infty}\left(X ; M_{N}(\mathbb{C})\right) \rightarrow C^{\infty}\left(X_{m} ; M_{N}(\mathbb{C})\right) \rightarrow 0
$$

is an admissible extension of Fréchet algebras. Using the finiteness of $G$ we see that $I_{m}^{G}$ is an admissible ideal in $I_{m+1}^{G}$ and that

$$
I_{m+1}^{G} / I_{m}^{G} \cong\left(I_{m+1} / I_{m}\right)^{G} \cong C_{0}^{\infty}\left(X_{m}, X_{m+1} ; M_{N}(\mathbb{C})\right)^{G}
$$


In order to apply Lemma 4 to the sequence

$$
0=I_{0}^{G} \subset I_{1}^{G} \subset \cdots \subset I_{\operatorname{dim} X}^{G} \subset I_{1+\operatorname{dim} X}^{G}=A^{G}
$$

we only have to show that the algebras in (23) are in the category $\mathcal{C}$. In fact, since $\overline{U_{\sigma} \backslash D_{\sigma}} \cap$ $\overline{U_{\tau} \backslash D_{\tau}}=\emptyset$ if $\operatorname{dim} \sigma=\operatorname{dim} \tau$ and $\sigma \neq \tau$, we have an isomorphism

$$
I_{m+1} / I_{m} \cong \prod_{m+\operatorname{dim} \sigma=\operatorname{dim} X} C_{0}^{\infty}\left(U_{\sigma}, D_{\sigma} ; M_{N}(\mathbb{C})\right)
$$

Now $G$ permutes the simplices in this product, so

$$
I_{m+1}^{G} / I_{m}^{G} \cong \prod_{\sigma \in L_{m}} C_{0}^{\infty}\left(U_{\sigma}, D_{\sigma} ; M_{N}(\mathbb{C})\right)^{G_{\sigma}}
$$

where $L_{m}$ is a set of representatives of the simplices of dimension $\operatorname{dim} X-m$, modulo the action of $G$. Invoking the additivity of $K_{*}$ and $H P_{*}$ we reduce our task to verifying that every factor of (26) belongs to $\mathcal{C}$. (This is somewhat problematic! See the appendix.)

If $m=\operatorname{dim} X$ then $D_{\sigma}$ is empty and we see from Lemma 7 that $C^{\infty}\left(U_{\sigma} ; M_{N}(\mathbb{C})\right)^{G_{\sigma}}$ has the required property.

For smaller $m$ there also exists (for every $\sigma$ ) a $G_{\sigma}$-equivariant contraction of $U_{\sigma}$ to a point $x_{\sigma} \in \psi(\sigma)$. Thus we can follow the proof of Lemma 7 up to equation (20), where we find that the factor of (26) corresponding to $\sigma$ is isomorphic to $C_{0}^{\infty}\left(U_{\sigma}, D_{\sigma} ; \operatorname{End}_{\mathbb{C}}\left(V_{\sigma}\right)\right)^{G_{\sigma}}$. Here $\left(\pi_{\sigma}, V_{\sigma}\right)$ denotes the projective $G_{\sigma}$-representation over the point $x_{\sigma}$. Using the $G_{\sigma}$-equivariant retraction of $U_{\sigma} \backslash D_{\sigma}$ to $\psi\left(\sigma \backslash D_{\sigma}^{\prime}\right)$ we see that this algebra is diffeotopy-equivalent to $C_{0}^{\infty}\left(\sigma, \sigma \cap D_{\sigma}^{\prime}\right) \otimes \operatorname{End}_{G_{\sigma}}\left(V_{\sigma}\right)$. The right hand side of this tensor product has finite dimension and is semisimple, so by the stability of $\mathcal{C}$ it presents no problems. Seen from its barycenter $\sigma \backslash D_{\sigma}^{\prime}$ is star-shaped, hence by Lemma 8 the left hand side is also in the category $\mathcal{C}$.

We conclude that all the algebras in (23) and (26) are indeed objects of $\mathcal{C}$, so Lemma 4 can be applied to (24) to prove that $A^{G} \in \mathrm{Ob}(\mathcal{C})$.

Note that the simplicial complex $\Sigma$ has only finitely many vertices if $X$ is compact, so then all the above direct products are in fact finite and $K_{*}\left(A^{G}\right)$ is finitely generated.

It is clear from the proofs of Lemma 7 and Theorem 6 that many similar Fréchet algebras are also in $\mathcal{C}$. For example if $Y$ is a closed submanifold of $X$ then the algebra

$$
B=\left\{f \in C^{\infty}\left(X ; M_{2}(\mathbb{C})\right): f(y) \text { diagonal } \forall y \in Y\right\}
$$

is in $\mathcal{C}$, as can be seen from the admissible extension

$$
0 \rightarrow C_{0}^{\infty}\left(X, Y ; M_{2}(\mathbb{C})\right) \rightarrow B \rightarrow C^{\infty}(Y)^{2} \rightarrow 0
$$

The algebra $A$ of Theorem [6 is a finitely generated module over $C^{\infty}(X)^{G}=C^{\infty}(X / G)$, so more generally one might consider Fréchet algebras that are finitely generated modules over $C^{\infty}(Y)$ with $Y$ an orbifold. The only real problem to generalizing our method to such algebras seems to be that Lemma 7 does not apply automatically, so we need a stronger kind of Poincaré lemma. This would require a detailed study of the type of algebras that can arise in this way.

Now we give some examples of Fréchet algebras to which Theorem [6] definitely applies.

Corollary 9. Let $\mathcal{H}$ be an affine Hecke algebra. Its Schwartz completion $\mathcal{S}(\mathcal{H})$ belongs to $\mathcal{C}$ and has finitely generated $K$-groups.

Proof. In Theorem 4.3 of [DO] Delorme and Opdam established an isomorphism between $\mathcal{S}(\mathcal{H})$ and a finite direct sum of algebras of the type described in Theorem 6 , the $X$ in each summand being a compact torus. 
Let $G$ be a reductive $p$-adic group. Recall that for a compact open subgroup $K$ the Schwartz algebra $\mathcal{S}(G / / K)$ consists of all smooth rapidly decreasing $K$-biinvariant complex valued functions on $G$. The convolution product makes it a nuclear Fréchet algebra. The Schwartz algebra $\mathcal{S}(G)$ of $G$ is by definition the union over all compact open subgroups $\bigcup_{K} \mathcal{S}(G / / K)$, endowed with the inductive limit topology. It is a complete locally convex algebra and a nuclear vector space, but it is not metrizable.

Furthermore $\mathcal{H}(G / / K)$ is the subalgebra of $\mathcal{S}(G / / K)$ consisting of compactly supported functions, and $\mathcal{H}(G):=\bigcup_{K} \mathcal{H}(G / / K)$ is called the Hecke algebra of $G$. These subalgebras are not complete, and their homologies are usually studied with respect to the algebraic tensor product.

Having introduced these objects, we state a crudely simplified version of Harish-Chandra's Plancherel formula for $p$-adic groups $[\mathrm{HC}$, a full proof of which was supplied by Waldspurger Wal.

Theorem 10. There exists a countable collection of triples $\left(T_{n}, L_{n}, \Gamma_{n}\right)$ with the following properties. For every $n T_{n}$ is a compact torus, $\Gamma_{n}$ a finite group acting on $T_{n}$ through diffeomorphisms $\alpha_{\gamma}$ and $L_{n}$ an algebra of bounded operators on a Hilbert space. There is a $G \times G$-action on $L_{n}$ such that for any compact open subgroup $K$ of $G$ the invariant algebra $L_{n}^{K \times K}$ is semisimple and has finite dimension. The group $\Gamma_{n}$ acts on $C^{\infty}\left(T_{n} ; L_{n}\right)$ by

$$
\gamma \cdot f(x)=c_{\gamma}(x) f\left(\alpha_{\gamma}^{-1} x\right)
$$

where $c_{\gamma}(x) \in \operatorname{Aut}_{G \times G} L_{n}$. All this results in an isomorphism

$$
\mathcal{S}(G) \cong \bigoplus_{n=1}^{\infty} C^{\infty}\left(T_{n} ; L_{n}\right)^{\Gamma_{n}}
$$

where the right hand side has the inductive limit topology with respect to the direct sum and the $L_{n}^{K \times K}$. Furthermore, for every $K$ there exist suitable numbers $n_{1}, \ldots, n_{N_{K}}$ such that the restriction to $K$-biinvariants is an isomorphism

$$
\mathcal{S}(G / / K) \cong \bigoplus_{i=1}^{N_{K}} C^{\infty}\left(T_{n_{i}} ; L_{n_{i}}^{K \times K}\right)^{\Gamma_{n_{i}}}
$$

For this action of $\Gamma_{n_{i}}$ there are $u_{\gamma} \in C^{\infty}\left(T_{n_{i}} ; L_{n_{i}}^{H \times H}\right)$ such that

$$
\gamma \cdot f(x)=u_{\gamma}(x) f\left(\alpha_{\gamma}^{-1} x\right) u_{\gamma}^{-1}(x)
$$

Proof. We will show that deriving this theorem from Wal] is merely a matter of translating. We will freely use Waldpurger's notation, which unfortunately differs substantially from ours, and we start by noticing that he writes $\mathcal{C}(G)$, respectively $\mathcal{C}_{H}$, for what we call $\mathcal{S}(G)$, respectively $\mathcal{S}(G / / H)$. The right hand side of (28) is

$$
C^{\infty}(\Theta)^{\mathrm{inv}}=\left(\bigoplus C^{\infty}(\mathcal{O}, P)\right)^{W^{G}}
$$

The direct sum runs over all parabolic subgroups $P$ of $G$ that contain a certain fixed maximal torus $A_{0}$. Let $P=M U$ be the Levi decomposition such that $A_{0} \subset M$ and denote the compact torus of unitary characters of $M$ by $\operatorname{Im} X(M)$. Let $(\omega, E)$ be an irreducible square-integrable admissible representation of $M$ and construct

$$
L(\omega, P):=I_{P \times P}^{G \times G}(E \otimes \breve{E})=\bigcup_{H<G} L(\omega, P)^{H \times H}
$$

where $I$ denotes induction with respect to compactly supported smooth functions, $\breve{E}$ is the contragredient representation of $E$ and we take the union over all compact open subgroups $H$ of $G$. 
By the admissibility of $E$ each $L(\omega, P)^{H \times H}$ has finite dimension, and it is a *-algebra since we can simply transfer the ${ }^{*}$ from $\mathcal{S}(G / / H)$. In particular it is semisimple.

All this leads to the identification

$$
C^{\infty}(\mathcal{O}, P)=\left(C^{\infty}(\operatorname{Im} X(M)) \otimes_{\mathbb{C}} L(\omega, P)\right)^{\operatorname{Stab}_{\operatorname{Im} X(M)}(\omega)}
$$

where the indicated stabilizer acts as in (27), $\alpha_{\gamma}$ being translation by $\gamma$. Likewise, the Weyl group $W^{G}$ acts on $C^{\infty}(\Theta)$ as in (27), where we should read ${ }^{\circ} c_{P^{\prime} \mid P}(\gamma, x)$ for $c_{\gamma}(x)$. Of course this doesn't fix the $P$ 's and the $M$ 's, so the $c_{\gamma}(x)$ live in something like $\operatorname{Hom}_{G \times G}\left(L_{n}, L_{m}\right)$. In section V.3 it is shown that these intertwiners are smooth in $x$. Now we take representatives for the association classes of the action of $W^{G}$ on the components of $\Theta$, so that $T_{n}=\operatorname{Im} X(M), L_{n}=L(\omega, P)$ and $\Gamma_{n}$ is semidirect product of the stabilizers of $\omega$ in $\operatorname{Im} X(M)$ and of $M$ in $W^{G}$.

This completes the translation of (28) to Théorèmes VII.2.5 and VIII.1.1 of WWal, leaving (29) and (30), which are not stated explicitly in that paper.

For an arbitrary compact open subgroup $H$ we consider the characteristic function $e_{H} \in$ $\mathcal{S}(G / / H)$ defined by

$$
e_{H}(g)=\left\{\begin{array}{lll}
0 & \text { if } & g \notin H \\
\operatorname{mes}(H)^{-1} & \text { if } & g \in H
\end{array}\right.
$$

It is an idempotent and its image under (28) lives only in those components for which $L(\omega, P)^{H \times H} \neq$ 0 . These are finite in number and we label them by $n_{1}, \ldots, n_{N_{H}}$. Since

$$
e_{H} \mathcal{S}(G) e_{H}=\mathcal{S}(G / / H)=\mathcal{S}(G)^{H \times H}
$$

and the actions of $\Gamma_{n}$ and $H \times H$ commute, we get (29). Moreover as $\operatorname{Aut}\left(M_{N}(\mathbb{C})\right)=P G L\left(\mathbb{C}^{N}\right)$, the automorphism $c_{\gamma}(x)$ of $L_{n_{i}}^{H \times H}$ is in fact conjugation by an invertible element $u_{\gamma}(x) \in L_{n_{i}}^{H \times H}$ and, $c_{\gamma}$ being smooth, we can arrange that $u_{\gamma} \in C^{\infty}\left(T_{n_{i}} ; L_{n_{i}}^{H \times H}\right)$.

Corollary 11. $\mathcal{S}(G / / K) \in \mathrm{Ob}(\mathcal{C})$ and its $K$-theory is finitely generated.

This corollary provides the small last step needed to complete the proof of a conjecture of Baum, Higson and Plymen. Confer BHP, in particular 8.9 and 9.4, for more background.

Theorem 12. Let $X$ and $C_{r}^{*}(G)$ be respectively the affine Bruhat-Tits building and the reduced $C^{*}$-algebra of $G$. There exists a commutative diagram

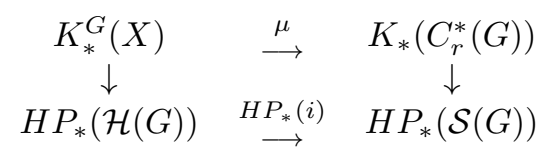

Here the vertical arrows are Chern characters, $\mu$ is the Baum-Connes assembly map and $H P_{*}(i)$ is induced by the inclusion $i: \mathcal{H}(G) \rightarrow \mathcal{S}(G)$. The horizontal maps are isomorphisms and the vertical maps become isomorphisms after tensoring the diagram with $\mathbb{C}$.

Proof. In Laf] Lafforgue proved the Baum-Connes conjecture for reductive $p$-adic groups, which is another way of saying that $\mu$ is an isomorphism. The commutativity of the diagram and the statement on the left vertical map can be found in BHP. Recall that $\mathcal{S}(G)=\lim _{\longrightarrow} \mathcal{S}(G / / K)$ is a holomorphically closed dense subalgebra of $C_{r}^{*}(G)$. Hence from the density theorem and the continuity of the $K$-functor we get

$$
K_{*}\left(C_{r}^{*}(G)\right) \cong \lim _{\longrightarrow} K_{*}(\mathcal{S}(G / / K))
$$

To avoid possible problems with the continuity of periodic cyclic homology, in BHP $H P_{*}(\mathcal{S}(G))$ is defined as $\lim _{\longrightarrow} H P_{*}(\mathcal{S}(G / / K))$. Now Corollary 11 says that the right vertical map becomes an 
isomorphism after tensoring with $\mathbb{C}$, so that the entire diagram will then consist of isomorphic objects. Thus $H P_{*}(i)$, being unmodified by this tensoring, is also an isomorphism.

Actually the formulation of Theorem 12 is somewhat imprecise since, after specifying a particular topological tensor product, we might also calculate $H P_{*}(\mathcal{S}(G))$ directly. (Well, in theory at least.) Because $S(G)$ is an inductive limit, it is best to use the inductive tensor product. If the result would be isomorphic to $\lim _{\longrightarrow} H P_{*}(\mathcal{S}(G / / K))$, which does not seem unlikely, then the theorem has a better and stronger meaning.

Indeed for $G=G L(m, F)$, with $F$ a non-Archimedean local field, Theorem 12 was already proved by Brodzki and Plymen [BP2], and they also showed in BP1] that

$$
H P_{*}(\mathcal{S}(G)) \cong \lim _{\longrightarrow} H P_{*}(\mathcal{S}(G / / K))
$$

However there are obstacles to generalizing this result to other reductive $p$-adic groups. Namely, the proof depends on the vanishing of the topological Hochschild homology groups

$H H_{n}(\mathcal{S}(G L(m, F) / / K))$ for all $n$ larger then a certain number, independent of $K$. To show this one uses that $\mathcal{S}(G L(m, F) / / K)$ is Morita-equivalent to a finite direct sum of commutative Fréchet algebras of the type $C^{\infty}(X)^{W}$, for suitable $X$ and $W$. This is a specific property of $G L(m, F)$ which does not hold for all other groups.

On the other hand, Nistor [Nis2] showed that for any reductive $p$-adic group $H H_{n}(\mathcal{H}(G))=0$ if $n$ exceeds the split rank of $G$. By the continuity of algebraic Hochschild homology this means that $H H_{n}(\mathcal{H}(G / / K))$ will vanish for such $n$, for a cofinal collection of compact open subgroups $K$. Although Nistor's techniques do not seem to carry over to the Schwartz completions, it is not unreasonable to expect that $H H_{n}(\mathcal{S}(G / / K))$ also vanishes for large $n$. Using [BP1] this would imply (31).

Because of (12) one might even hope that for all the algebras of Theorem 6 we have $H H_{n}\left(A^{G}\right)=$ 0 if $n$ exceeds some bound which depends only on $G$ and $\operatorname{dim} X$. Using the proof of Theorem 6 we can reduce this problem to the algebras appearing in equation (26) but there the applicability of this paper ends, since Lemmas 7 and 8 both rely in an essential way on the diffeotopy-invariance of $K_{*}$ and $H P_{*}$, a property that $H H_{*}$ does not possess.

Finally we remark that recently Meyer Mey1 studied the inclusion $\mathcal{H}(G) \rightarrow \mathcal{S}(G)$ from a different perspective, namely that of representations of bornological algebras. He obtained strong results on the comparison of the categories of tempered representations of these algebras. It is unclear to the author whether this has implications for the induced map on periodic cyclic homology. 


\section{Appendix, added September 2008}

Recently the author became aware that infinite direct products do not commute with algebraic tensor products. For example, $\left(\prod_{n=1}^{\infty} \mathbb{Z}\right) \otimes_{\mathbb{Z}} \mathbb{C}$ is strictly smaller than $\prod_{n=1}^{\infty} \mathbb{C}$. Therefore the map

$$
\text { ch } \otimes \mathrm{id}: K_{*}\left(\prod_{n=1}^{\infty} \mathbb{C}\right) \otimes_{\mathbb{Z}} \mathbb{C} \rightarrow H P_{*}\left(\prod_{n=1}^{\infty} \mathbb{C}\right)
$$

is injective, but fails to be a surjection. Hence the category $\mathcal{C}$ cannot be closed with respect to infinite direct products.

Unfortunately, this very assumption was used in the lines directly following (26). Rather than rewriting a part of the paper, the author decided to add this appendix, which discusses two ways to overcome the problem.

Firstly, we can avoid it altogether by restricting ourselves to algebras $A$ for which $H_{*}(A)$ has finite dimension. In the context of Theorem [6, this means that we have to require that the $G$-manifold $X$ admits a finite open cover, such that every intersection of elements of this cover is $G$-equivariantly contractible. This is the case for compact manifolds, and for algebraic varieties on which $G$ acts algebraically.

The second solution is more involved, and replaces $\mathcal{C}$ by a somewhat different class of algebras. The underlying idea is that $\prod_{n=1}^{\infty} \mathbb{C}$ and $\left(\prod_{n=1}^{\infty} \mathbb{Z}\right) \otimes_{\mathbb{Z}} \mathbb{C}$ are both nondegenerately paired with $\bigoplus_{n=1}^{\infty} \mathbb{C}$, and that this property is actually good enough. To make sense of this for general $\mathrm{m}$ algebras, we use the cohomology theories that are dual to $K$-theory and periodic cyclic homology.

Recall that Cuntz and Quillen CQ1, Section 10] defined a bivariant functor $H P_{*}(A, B)$, such that $H P_{*}(\mathbb{C}, B)=H P_{*}(B)$ and $H P_{*}(A, \mathbb{C})=H P^{*}(A)$, where $H P^{*}$ denotes periodic cyclic cohomology. The construction of bivariant periodic cyclic homology can be carried out in various categories of algebras, in particular for $m$-algebras. This functor is stable and diffeotopy-invariant in both variables [CQ2 , Section 3]. The excision property holds with respect to admissible extensions, also in both variables Cun1, Section 5]. There is a natural product

$$
H P_{*}(A, B) \times H P_{*}(B, D) \rightarrow H P_{*}(A, D),
$$

which in particular provides a bilinear form

$$
H P_{i}(A) \times H P^{i}(A) \rightarrow H P_{0}(\mathbb{C}, \mathbb{C}) \cong \mathbb{C} .
$$

Moreover Cuntz Cun2] developed a bivariant $k k$-theory for $m$-algebras, whose construction we briefly recall. Let $T A$ be the "projective" completion of the tensor algebra of $A$, and define $J A$ as the kernel of the multiplication map $T A \rightarrow A$. Let $\mathfrak{K}$ be the $m$-algebra of infinite matrices with rapidly decaying coefficients, which is embedded in usual $C^{*}$-algebra of compact operators. Denote the collection of homotopy classes of $m$-algebra homomorphisms $A \rightarrow B$ by $\langle A, B\rangle$. There exist natural maps $\left\langle J^{n} A, B\right\rangle \rightarrow\left\langle J^{n+2} A, \mathfrak{K} \widehat{\otimes} B\right\rangle$, where $\widehat{\otimes}$ denotes the completed projective tensor product. Cuntz defines

$$
k k_{j}(A, B)=\lim _{n \rightarrow \infty}\left\langle J^{2 n+j} A, \mathfrak{K} \widehat{\otimes} B\right\rangle .
$$

This theory takes values in abelian groups and is 2-periodic. Moreover $k k_{*}$ satisfies all the formal properties of $H P_{*}$ which we described above. We write

$$
K_{*}(A)=k k_{*}(\mathbb{C}, A) \quad \text { and } \quad K^{*}(A)=k k_{*}(A, \mathbb{C}) .
$$

This agrees with the classical $K$-theory for Banach algebras, and with Phillips' Phi] $K$-theory for Fréchet algebras. However, it differs from Kasparov's $K K$-theory and from $K$-homology for separable $C^{*}$-algebras. The product in $k k$-theory gives a $\mathbb{Z}$-bilinear form

$$
K_{i}(A) \times K^{i}(A) \rightarrow k k_{0}(\mathbb{C}, \mathbb{C}) \cong \mathbb{Z} .
$$


Finally, there exists a functorial bivariant Chern character

$$
c h: k k_{*}(A, B) \rightarrow H P_{*}(A, B),
$$

which is compatible with all these properties.

In general $k k_{*}\left(\prod_{n=1}^{\infty} A_{n}, \prod_{m=1}^{\infty} B_{m}\right)$ is neither isomorphic to $\prod_{m=1}^{\infty} k k_{*}\left(\prod_{n=1}^{\infty} A_{n}, B_{m}\right)$, nor to $\bigoplus_{n=1}^{\infty} k k_{*}\left(A_{n}, \prod_{m=1}^{\infty} B_{m}\right)$. For example, the identity $\operatorname{map} \operatorname{id}_{P}$ of $P=\prod_{n=1}^{\infty} \mathbb{C}$ cannot be represented by an element of $\bigoplus_{n=1}^{\infty} k k_{*}(\mathbb{C}, P)$. Similar phenomena occur in bivariant periodic cyclic homology. Fortunately the univariant versions of these functors do behave well with respect to infinite direct products:

Proposition A.13. Let $A_{i}(i \in I)$ be an arbitrary collection of $m$-algebras. There are natural isomorphisms

$$
\begin{aligned}
& H P_{*}\left(\prod_{i \in I} A_{i}\right) \cong \prod_{i \in I} H P_{*}\left(A_{i}\right), \\
& H P^{*}\left(\prod_{i \in I} A_{i}\right) \cong \bigoplus_{i \in I} H P^{*}\left(A_{i}\right), \\
& K_{*}\left(\prod_{i \in I} A_{i}\right) \cong \prod_{i \in I} K_{*}\left(A_{i}\right), \\
& K^{*}\left(\prod_{i \in I} A_{i}\right) \cong \bigoplus_{i \in I} K^{*}\left(A_{i}\right) .
\end{aligned}
$$

Proof. Let $B$ be any $m$-algebra and abbreviate $A=\prod_{i \in I} A_{i}$.

By definition $H P_{*}(B)$ is the homology of a differential complex $\mathcal{X}(\overleftarrow{T} B)$, see [Mey2, Section 4.1.5]. According to Mey2, Theorem 4.3.7] the canonical map

$$
\mathcal{X}(\overleftarrow{T} A) \rightarrow \prod_{i \in I} \mathcal{X}\left(\overleftarrow{T} A_{i}\right)
$$

is a homotopy equivalence, which leads to A.35).

Furthermore $H P^{*}(B)$ is the cohomology of $\operatorname{Hom}(\mathcal{X}(\overleftarrow{T} B), \mathbb{C})$, where we must take the homomorphisms in the category of projective limits of Banach spaces. Recall that the continuous linear dual of a direct product of topological vector spaces can be identified with the direct sum of the dual spaces. Together with (A.39) we find homotopy equivalences

$$
\operatorname{Hom}(\mathcal{X}(\overleftarrow{T} A), \mathbb{C}) \leftarrow \operatorname{Hom}\left(\prod_{i \in I} \mathcal{X}\left(\overleftarrow{T} A_{i}\right), \mathbb{C}\right) \cong \bigoplus_{i \in I} \operatorname{Hom}\left(\mathcal{X}\left(\overleftarrow{T} A_{i}\right), \mathbb{C}\right)
$$

which implies (A.36).

From [Cun2, p. 178] we see that

$$
\begin{aligned}
& K_{0}(B)=k k_{0}(\mathbb{C}, B) \cong\langle q \mathbb{C}, \mathfrak{K} \widehat{\otimes} B\rangle \\
& K_{1}(B)=k k_{1}(\mathbb{C}, B) \cong\langle q \mathbb{C}, \mathfrak{K} \widehat{\otimes} \mathbb{C}(0,1) \widehat{\otimes} B\rangle,
\end{aligned}
$$

where the Fréchet algebras $q \mathbb{C}$ and $\mathbb{C}(0,1)$ are as in Cun2, Section 1]. Using the compatibility of $\widehat{\otimes}$ with direct products [Gro1, Proposition I.1.3.6] we get natural isomorphisms

$$
K_{0}\left(\prod_{i \in I} A_{i}\right) \cong\left\langle q \mathbb{C}, \prod_{i \in I}\left(\mathfrak{K} \widehat{\otimes} A_{i}\right)\right\rangle \cong \prod_{i \in I}\left\langle q \mathbb{C}, \mathfrak{K} \widehat{\otimes} A_{i}\right\rangle \cong \prod_{i \in I} K_{0}\left(A_{i}\right) .
$$

The same goes for $K_{1}(A)$, proving A.37).

On the other hand, for $B=\mathbb{C}$ A.33 becomes

$$
K^{j}(A)=k k_{j}(A, \mathbb{C})=\lim _{n \rightarrow \infty}\left\langle J^{2 n+j} A, \mathfrak{K}\right\rangle .
$$

For any finite subset $F \subset I$ we write $A_{F}=\bigoplus_{i \in F} A_{i}$. Since $k k_{*}$ satisfies excision,

$$
K^{*}\left(A_{F}\right) \cong \bigoplus_{i \in F} K^{*}\left(A_{i}\right) \text {. }
$$


The inclusion and quotient maps $A_{F} \rightarrow A \rightarrow A_{F}$ induce group homomorphisms

$$
K^{*}\left(A_{F}\right) \rightarrow K^{*}(A) \rightarrow K^{*}\left(A_{F}\right)
$$

whose composition is the identity. These combine to a natural injection

$$
\bigoplus_{i \in I} K^{*}\left(A_{i}\right) \rightarrow K^{*}(A) .
$$

Consider the following subalgebra of $J^{m}(A)$ :

$$
\left(J^{m} A\right)_{F}:=J^{m}\left(A_{F}\right) \cap \bigcap_{i \in F} \operatorname{ker}\left(J^{m}\left(A_{F}\right) \rightarrow J^{m}\left(A_{F \backslash\{i\}}\right)\right) .
$$

It can be described as the subspace of $J^{m}(A)$ which is spanned by all tensors which only involve elements from the $A_{i}$ with $i \in F$, and which is complementary to the tensors coming from fewer summands $A_{i}$. Notice that

$$
J^{m}\left(A_{F}\right)=\prod_{F^{\prime} \subset F}\left(J^{m} A\right)_{F^{\prime}} .
$$

This product is direct in the category of topological vector spaces, but as a product of algebras it is only semi-direct. Since $J^{m} A=\lim _{F} J^{m}\left(A_{F}\right)$, we can identify it as a topological vector space with the direct product of the $\left(J^{m} A\right)_{F}$, over all finite subsets $F$ of $I$ :

$$
J^{m}(A)=\prod_{F}\left(J^{m} A\right)_{F} .
$$

To show that (A.40) is surjective, take any class $[f] \in K^{j}(A)$, represented by an $m$-algebra homomorphism $f: J^{2 n+j} A \rightarrow \mathfrak{K}$. Let $\|$ ? $\|_{o}$ be operator norm on the pre- $C^{*}$-algebra $\mathfrak{K}$, so that

$$
J^{2 n+j} A \rightarrow \mathbb{R}: a \mapsto\|f(a)\|_{o}
$$

is a continuous map. Thus

$$
U:=\left\{a \in J^{2 n+j} A:\|f(a)\|_{o}<1\right\}
$$

is open. Since the right hand side of (A.41) is equipped with the product topology, we have $\left(J^{2 n+j} A\right)_{F} \subset U$ for all but finitely many $F$. Clearly this implies $f\left(\left(J^{2 n+j} A\right)_{F}\right)=0$ for these $F \subset I$. Let $I_{f}$ be the union of the $F \subset I$ for which $f\left(\left(J^{2 n+j} A\right)_{F}\right) \neq 0$. Then $I_{f}$ is finite and $f$ factors as

$$
J^{2 n+j}(A) \rightarrow J^{2 n+j}\left(A_{I_{f}}\right) \rightarrow \mathfrak{K} .
$$

This means that $[f]$ lies in the image of $K^{j}\left(A_{I_{f}}\right) \rightarrow K^{j}(A)$, so (A.40) is indeed surjective.

In many cases the pairings A.32 and A.34 are nondegenerate, so it makes sense to consider the following class of algebras.

Definition A.14. The class $\mathcal{C}^{\prime}$ consists of all $m$-algebras A satisfying the following conditions:

1. $H P_{*}(A) \times H P^{*}(A) \rightarrow \mathbb{C}$ is a nondegenerate bilinear pairing,

2. ch: $K^{*}(A) \otimes_{\mathbb{Z}} \mathbb{C} \rightarrow H P^{*}(A)$ is a linear bijection,

3. $K_{*}(A) \otimes_{\mathbb{Z}} \mathbb{C} \times K^{*}(A) \otimes_{\mathbb{Z}} \mathbb{C} \rightarrow \mathbb{C}$ is a nondegenerate bilinear pairing.

For any $m$-algebra $A$ in $\mathcal{C}^{\prime}$, the Chern character

$$
\text { ch }: K_{*}(A) \otimes_{\mathbb{Z}} \mathbb{C} \rightarrow H P_{*}(A)
$$


is injective and has dense image, with respect to the coarsest topology on $H P_{*}(A)$ that makes all elements of $H P^{*}(A)$ into continuous linear functionals. In particular, if $H P_{*}(A)$ has finite dimension, then (A.42) is a linear bijection and $A$ also belongs to $\mathcal{C}$.

From the above discussion we already know that $\mathcal{C}^{\prime}$ is closed under diffeotopy equivalences and under tensoring with $M_{k}(\mathbb{C})$. Proposition $\AA .13$ and the compatibility of $\otimes_{\mathbb{Z}} \mathbb{C}$ with direct sums show that $\mathcal{C}^{\prime}$ is closed with respect to arbitrarily large direct products.

After these remarks we will show that $\mathcal{C}^{\prime}$ has the same "two out of three"-property as $\mathcal{C}$. Hence $\mathcal{C}^{\prime}$ really has all the properties claimed for $\mathcal{C}$ in Corollary 3. Since these are all that is used in the remainder of the paper, we conclude that all the results, in particular Theorem 6 , hold if we replace $\mathcal{C}$ by $\mathcal{C}^{\prime}$. Moreover Corollaries 9 and 11 are valid precisely as stated (with $\mathcal{C}$ ), because the periodic cyclic homology has finite dimension in these cases.

Lemma A.15. Let $0 \rightarrow A \stackrel{\phi}{\rightarrow} B \stackrel{\psi}{\rightarrow} D \rightarrow 0$ be an admissible extension of $m$-algebras. If two elements of $\{A, B, D\}$ belong to $\mathcal{C}^{\prime}$, then so does the third.

Proof. Of the three conditions in Definition A.14, we can handle the second just as in the proof of Corollary 3. As moreover all cases of the first and third conditions can be dealt with in the same way, we will only treat one case. Suppose that $A$ and $D$ belong to $\mathcal{C}^{\prime}$. We want to show that $H P_{0}(B) \times H P^{0}(B) \rightarrow \mathbb{C}$ is a nondegenerate bilinear pairing. Consider the exact sequences

$$
\begin{aligned}
& \cdots \rightarrow H P_{1}(D) \rightarrow H P_{0}(A) \rightarrow H P_{0}(B) \rightarrow H P_{0}(D) \rightarrow H P_{1}(A) \rightarrow \cdots \\
& \cdots \leftarrow H P^{1}(D) \leftarrow H P^{0}(A) \leftarrow H P^{0}(B) \leftarrow H P^{0}(D) \leftarrow H P^{1}(A) \leftarrow \cdots
\end{aligned}
$$

In every column there is a bilinear pairing, and every arrow is adjoint to the one directly below or above it. By assumption all these pairings are nondegenerate, except possibly in the middle column. Therefore

$$
\begin{array}{ll}
\operatorname{im}\left(H P_{1}(D)\right)^{\perp}=\operatorname{im}\left(H P^{0}(B)\right) & \operatorname{im}\left(H P_{0}(B)\right)^{\perp}=\operatorname{im}\left(H P^{1}(A)\right) \\
\operatorname{im}\left(H P_{1}(D)\right)=\operatorname{im}\left(H P^{0}(B)\right)^{\perp} & \operatorname{im}\left(H P_{0}(B)\right)=\operatorname{im}\left(H P^{1}(A)\right)^{\perp},
\end{array}
$$

and we can simplify the above diagram to

$$
\begin{aligned}
& 0 \rightarrow H P_{0}(A) / \operatorname{im}\left(H P_{1}(D)\right) \rightarrow H P_{0}(B) \rightarrow \quad \rightarrow \quad \operatorname{im}\left(H P_{0}(B)\right) \rightarrow 0 \\
& 0 \leftarrow \operatorname{im}\left(H P^{0}(B)\right) \leftarrow H P^{0}(B) \leftarrow H P^{0}(D) / \operatorname{im}\left(H P^{1}(A)\right) \leftarrow 0
\end{aligned}
$$

The rows remain exact and the second and fourth columns are endowed with nondegenerate bilinear pairings. Consider any $x \in H P_{0}(B) \cap H P^{0}(B)^{\perp}$. Then $H P_{0}(\psi)(x) \in H P^{0}(D)^{\perp}$, so by the nondegeneracy of

$$
\operatorname{im}\left(H P_{0}(B)\right) \times H P^{0}(D) / \operatorname{im}\left(H P^{1}(A)\right) \rightarrow \mathbb{C}
$$

we have $H P_{0}(\psi)(x)=0$. Thus $x=H P_{0}(\phi)(y)$ for some $y \in H P_{0}(A)$. But

$$
y \in \operatorname{im}\left(H P^{0}(B)\right)^{\perp}=\operatorname{im}\left(H P_{1}(D)\right), \text { so } x \in H P_{0}(\phi)\left(H P_{1}(D)\right)=0 .
$$

A similar argument shows that $H P^{0}(B) \cap H P_{0}(B)^{\perp}=0$.

\section{Acknowledgements.}

The author would like to thank Ralf Meyer, Victor Nistor, Eric Opdam and Peter Schneider for their helpful comments. 


\section{References}

[AH] M.F. Atiyah, F.E.P. Hirzebruch, "Vector bundles and homogeneous spaces", in: Differential geometry, 7-38, Proc. Sympos. Pure Math. 3, American Mathematical Society, Providence RI, 1961

[BC] P.F. Baum, A. Connes, "Chern character for discrete groups", in: A fête of topology, 163-232, Academic Press, Boston MA, 1988

[BHP] P.F. Baum, N. Higson, R.J. Plymen, "Representation theory of p-adic groups: a view from operator algebras", in: Proc. Sympos. Pure. Math. 68, 111-149, American Mathematical Society, Providence RI, 2000

[Bla] B. Blackadar, "K-theory for operator algebras 2nd ed.", Cambridge University Press, Cambridge, 1998

[BP1] J. Brodzki, R.J. Plymen, "Periodic cyclic homology of certain nuclear algebras", C.R. Acad. Sci. Paris 329 (1999), 671-676

[BP2] J. Brodzki, R.J. Plymen, "Chern character for the Schwartz algebra of p-adic GL(n)", Bull. London Math. Soc. 34.2 (2002), 219-228

[Bry] J.L. Brylinski, "Algebras associated with group actions and their homology", Brown University preprint, Providence RI, 1987

[BN] J.L. Brylinski, V. Nistor, "Cyclic cohomology of étale groupoids", K-Theory 8.4 (1994), 341-365

[Con] A. Connes, "Noncommutative differential geometry", Publ. Math. Inst. Hautes Études Sci. 62 (1985), 41-144

[Cun1] J.R. Cuntz, "Excision in periodic cyclic theory for topological algebras", in: Cyclic cohomology and noncommutative geometry, 43-53, Fields Inst. Commun. 17, American Mathematical Society, Providence RI, 1997

[Cun2] J.R. Cuntz, "Bivariante K-Theorie für lokalkonvexe Algebren und der Chern-ConnesCharakter", Doc. Math 2 (1997), 139-182

[CQ1] J.R. Cuntz, D. Quillen, "Cyclic homology and nonsingularity", J. Amer. Math. Soc. 8.2 (1995), 373-442

[CQ2] J.R. Cuntz, D. Quillen, "Excision in bivariant periodic cyclic cohomology", Inv. Math. 127 (1997), 67-98

[DO] P. Delorme, E.M. Opdam, "The Schwartz algebra of an affine Hecke algebra", arXiv:math.RT/0312517, 2004

[Gro1] A. Grothendieck, "Produits tensoriels topologiques et espaces nucléaires", Mem. Amer. Math. Soc. 16, American Mathematical Society, Providence RI, 1955

[Gro2] A. Grothendieck, "Sur quelques points d'algèbre homologique" Tôhoku Math. J. II 9 (1957), $119-221$

[HC] Harish-Chandra, "The Plancherel formula for reductive p-adic groups", in: Collected papers vol. IV, 353-367, Springer-Verlag, New York, 1984 
[Ill] S. Illman, "Smooth equivariant triangulations of G-manifolds for G a finite group", Math. Ann. 233 (1978), 199-220

[Laf] V. Lafforgue, "K-théorie bivariante pour les algèbres de Banach et conjecture de BaumConnes", Invent. Math. 149.1 (2002), 1-95

[Mey1] R. Meyer, "Homological algebra for Schwartz algebras of reductive p-adic groups", arXiv:math.RT/0501548, 2005

[Mey2] R. Meyer, Local and analytic cyclic homology, Tracts in Mathematics 3, European Mathematical Society Publishing House, 2007

[Nis1] V. Nistor, "Higher index theorems and the boundary map in cyclic cohomology", Doc. Math. 2 (1997), 263-295

[Nis2] V. Nistor, "Higher orbital integrals, Shalika germs, and the Hochschild homology of Hecke algebras", Int. J. Math. Math. Sci. 26.3 (2001), 129-160

[Phi] N.C. Phillips, "K-theory for Fréchet algebras", Int. J. Math. 2.1 (1991), 77-129

[Sch] F. Schur, "Über die Darstellung der endlichen Gruppen durch gebrochene linaere Subsitutionen", J. reine angew. Math. 127 (1904), 20-50

[Ros] J.M. Rosenberg, "Appendix to: "Crossed products of UHF algebras by product type actions" by O. Bratteli", Duke Math. J. 46.1 (1979), 25-26

[Tou] J.C. Tougeron, Idéaux de fonctions différentiables, Ergebnisse der Mathematik und ihrer Grenzgebiete 71, Springer-Verlag, Berlin, 1972

[Wal] J.-L. Waldspurger, "La formule de Plancherel pour les groupes p-adiques (d'après HarishChandra)", J. Inst. Math. Jussieu 2.2 (2003), 235-333

[Was1] A.J. Wassermann, "Une démonstration de la conjecture de Connes-Kasparov pour les groupes de Lie linéaires connexes réductifs", C.R. Acad. Sci. Paris 304 (1987), 559-562

[Was2] A.J. Wassermann, "Cyclic cohomology of algebras of smooth functions on orbifolds", in: Operator algebras and applications 1, 229-244, London Math. Soc. Lecture Note Ser. 135, Cambridge University Press, Cambridge, 1988 